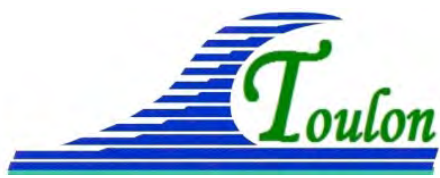

XIV èmes Journées Nationales Génie Côtier - Génie Civil

Toulon, 29 juin au $1^{\text {er }}$ juillet 2016

DOI:10.5150/jngcgc.2016.017 @ Editions Paralia CFL

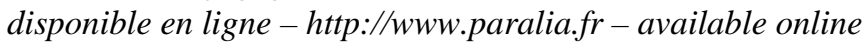

\title{
Soixante ans d'évolution de la flèche sableuse de Sables d'Or les Pins (Bretagne Nord)
}

\author{
Agnès BALTZER ${ }^{1}$, Chantal BONNOT ${ }^{2}$, \\ Nicolas ROLLO ${ }^{1}$, Bleuenn BODIN ${ }^{1}$, \\ Florian BERANGER ${ }^{2}$, Patrice BRETEL ${ }^{3}$, Lucile GLUARD ${ }^{3}$
}

1. Université de Nantes, LETG-Nantes Géolittomer, UMR 6554 CNRS - CNRS, Campus du Tertre, BP 81227, 44312 Nantes Cedex 3, France.

agnes.baltzer@univ-nantes.fr

2. Directeur de Recherche Honoraire CNRS.

3. Université de Caen, Faculté des Sciences, UMR 6143 CNRS - M2C, Esplanade de la Paix, 14032 Caen, France.

\section{Résumé :}

Le site de Sables d'Or les Pins constitue le seul exemple de flèche littorale sableuse à pointe libre de la côte orientale de la baie de Saint-Brieuc (Côtes d'Armor, Bretagne Nord) entre le Cap Fréhel et le Cap d'Erquy. Evoluant en régime macrotidal, cette flèche littorale est surmontée d'un important massif dunaire et isole à son extrémité Ouest une lagune côtière dans laquelle s'est développé un marais maritime. L’évolution des principales unités morpho-sédimentaires a été suivie grâce à l'analyse d'une chronique de photos aériennes couvrant ce secteur littoral et réparties sur une période allant de 1952 à 2012. Cette étude met en évidence les phases de progradation et/ou de recul du front de la végétation dunaire ainsi que l'évolution du schorre qui occupe les secteurs les plus abrités de la lagune. La dynamique active qui remanie les sables de l'extrémité de la flèche littorale peut être mise en relation avec les épisodes de tempêtes qui mobilisent le sable des barres sableuses de la plage externe.

Mots-clés : Flèche littorale sableuse, Massif dunaire, Régime macrotidal, Evolution pluri-décennale, Fréquence des tempêtes.

\section{Introduction}

L'objectif de cette étude est d'analyser l'évolution à long terme du système flèche littorale - massif dunaire - lagune côtière de Sables d'Or les Pins. La comparaison d'une chronique de photos aériennes s'étendant de 1952 à 2012 permet une cartographie précise de la frange littorale et conduit à une quantification de l'évolution du site. Cette étude devrait aider le département des Côtes d'Armor, qui souhaite prendre en compte les évolutions de la flèche dunaire, à établir un plan de gestion adapté à la dynamique de ce site. 


\section{Thème 2 - Dynamique sédimentaire}

\section{Cadre Général}

Le site de Sables d'Or constitue le seul exemple de flèche littorale sableuse à pointe libre de la côte orientale de la baie de Saint-Brieuc (figure 1a). Ce littoral coïncide avec un fossé d'effondrement limité par deux pointes rocheuses taillées dans les grès anciens de la formation d'Erquy -Fréhel. Un important massif dunaire surmonte le cordon littoral qui isole, à son extrémité Ouest, une lagune côtière dans laquelle débouche le ruisseau de l'Islet (BONNOT-COURTOIS \& LAFOND, 1994). Ce secteur côtier est soumis à un régime macrotidal avec un marnage variant de $7 \mathrm{~m}$ en morte-eau à 12,50 $\mathrm{m}$ en vive-eau (LCHF, 1982). Ouverte au Nord, la plage est relativement protégée des houles dominantes par la présence d'îlots (îlot Saint-Michel), de platiers rocheux et de pointes rocheuses (Pointe du Champ du Port) qui parsèment l'avant côte (figure 1b, BODIN, 2015). Ces houles de Nord-Ouest, de $H_{\max }=5 \mathrm{~m}$ (ALLEN, 1982) sont diffractées par ces obstacles de telle sorte qu'elles atteignent la partie Ouest de la plage avec une obliquité Nord/Nord-Est induisant une dérive littorale vers l'Ouest (PLUET \& ZIMMERMANN, 1984).
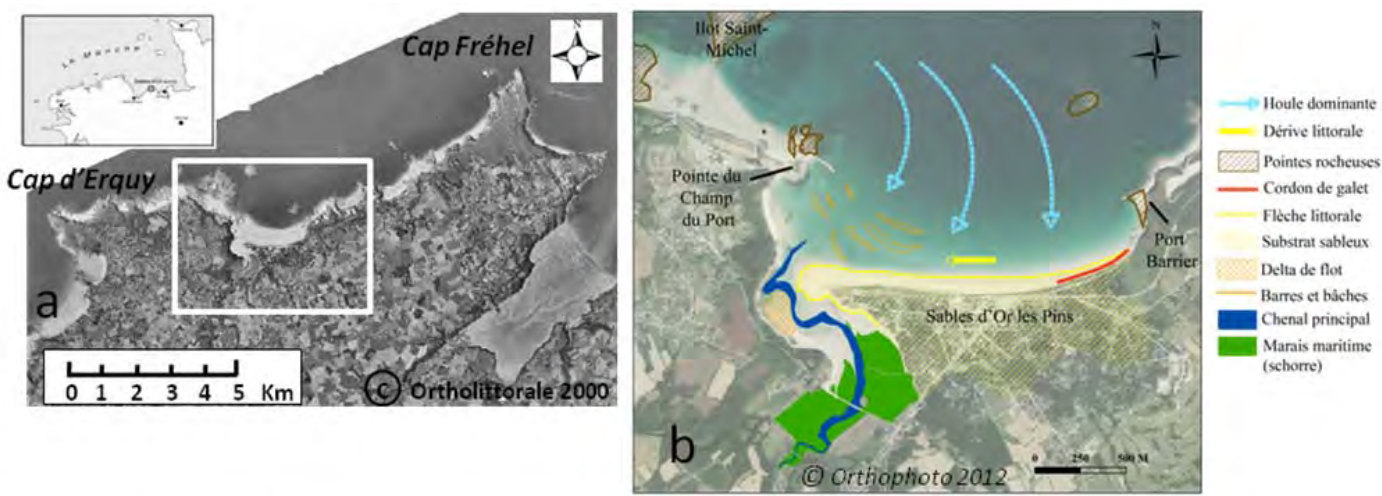

Figure 1. (a) Le littoral des Côtes d'Armor entre le Cap Fréhel et Erquy.

(b) Principales structures morpho-sédimentaires du littoral de Sables d'Or.

La partie orientale de la plage proche du Port Barrier, orientée face aux houles de NordOuest, non diffractées, est soumise à une forte agitation marquée par la présence d'un cordon de galets en haut de plage. Les houles de Nord atteignent frontalement la partie centrale de la plage qui a subi de fortes érosions jusqu'à un effondrement des cabines de bains sur la haute plage en 1990. Cet évènement est à l'origine de la mise en place en 1996 d'un enrochement pour protéger la route côtière (BONNOT-COURTOIS \& LANÇON, 2004). Dans la partie occidentale, de grandes rides péri-littorales occupent la basse et la moyenne plage. Orientées perpendiculairement aux orthogonales de houles diffractées, elles forment une succession de barres et de bâches qui se déplacent vers la haute plage. Elles alimentent la flèche littorale qui s'étend sur une longueur de $2 \mathrm{~km}$, depuis sa racine à l'Est vers Port Barrier jusqu'à son extrémité à pointe libre à l'Ouest (figure 1b). Les pleines mers de grande vive-eau atteignent le pied du massif dunaire qui 


\section{XIVìmes Journées Nationales Génie Côtier - Génie Civil \\ Toulon, 29 juin au $1^{\text {er }}$ juillet 2016}

surmonte la flèche littorale. Elle est surmontée par un très important massif dunaire qui a été largement arasé pour la construction de la station balnéaire dans les années 1920.

A son extrémité occidentale, la flèche sableuse a progressivement barré le fond de baie et isole en arrière d'elle une lagune côtière dans laquelle débouche le petit fleuve côtier de l'Islet. La progression de la flèche vers l'Ouest repousse le chenal contre la falaise des Hôpitaux mais la passe d'entrée reste entretenue par les forts courants de marée qui s'établissent lors du remplissage et de la vidange de la lagune. Les courants de flot transportent de grandes quantités de sables grossiers qui se dispersent à l'intérieur de la lagune sous forme d'un delta de flot, modelé par de nombreuses rides dont l'amplitude diminue progressivement depuis la passe d'entrée vers l'intérieur de la lagune. Ces sables très mobiles envahissent progressivement le revers interne de la flèche et recouvrent la bordure du schorre installé sur les sédiments fins vaseux qui occupent les secteurs les plus internes de la lagune.

\section{Evolution pluri-décennale du littoral}

L'évolution des unités morpho-sédimentaires qui constituent la partie Ouest du littoral de Sables d’Or a été étudiée à partir d'une série de photographies aériennes s'échelonnant entre 1952 et 2012, avec un intervalle d'années entre 2 prises de vue variant de 4 à 14 ans. Chaque cliché a été rectifié géométriquement et géoréférencé à l'aide du logiciel Er Mapper, en utilisant l’Ortholittorale 2000 comme référentiel. Selon les années, les erreurs de redressement varient de $2,5 \mathrm{~m}$ à plus de $8 \mathrm{~m}$ en fonction de la qualité des prises de vues et du nombre possible de points de calage. Les années 1981 et 1993 où les décalages étaient les plus importants n’ont pas été retenues et l'incertitude moyenne sur les distances mesurées est de 4,8m (BERANGER, 2005). La cartographie de l'estran, réalisée sous SIG (ArcView et ArcGis) avec une échelle de numérisation au $1 / 2000^{\text {ème }}$, permet de quantifier les évolutions de l'extrémité de la flèche littorale sableuse, du massif dunaire et de l’intérieur de la lagune côtière.

\subsection{La flèche littorale}

La morphologie de la pointe libre de la flèche sableuse est contrainte d'une part par les courants de marée, qui se renforcent dans la passe d'entrée dans la lagune, et d'autre part par la dynamique des barres sableuses de la plage qui alimentent l'extrémité de la flèche. En 1952, l'extrémité de la flèche était relativement étroite et déportée vers le Nord par rapport à la dune végétalisée (figure 2) (BODIN, 2015). En 1966, on constate un étalement très net de l'extrémité de la flèche qui, en 1981, revient près de sa position initiale de 1952. Entre 1981 et 2002, la flèche s'étale d'environ 50 m vers l'Ouest, suivi par un recul équivalent vers l'Est de 2002 à 2012 où la forme globale de la pointe libre semble se raccourcir et s'étendre vers le Sud. En 1981, 1993 et 2002, les divagations du chenal de marée à l'intérieur de la lagune provoquent des érosions du revers interne de la flèche. 


\section{Thème 2 - Dynamique sédimentaire}

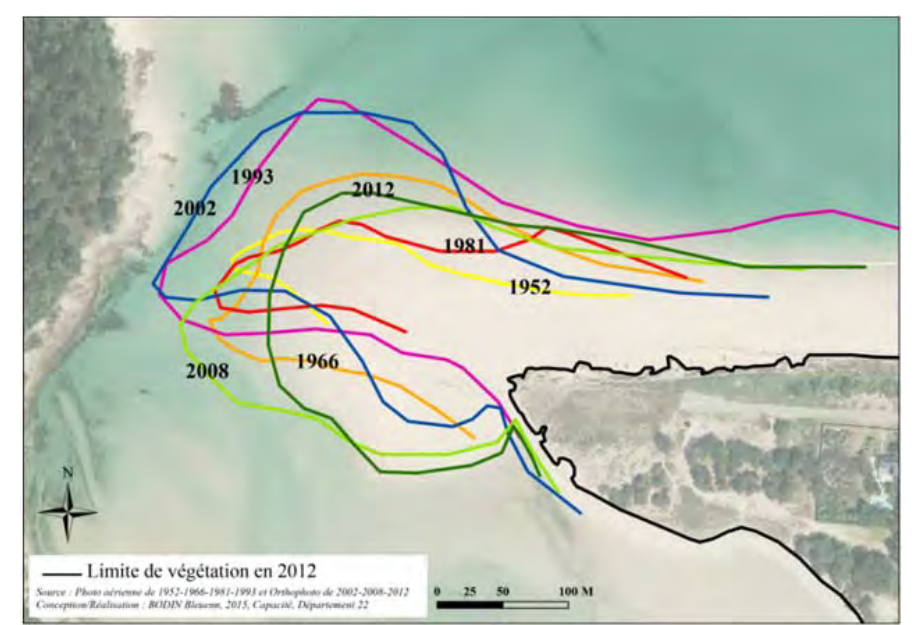

Figure 2. Evolution de l'extrémité de la flèche sableuse entre 1952 et 2012.

\subsection{Le massif dunaire}

La carte des Ingénieurs Géographes du Roi qui fait référence, par la précision de ses levés et la qualité de représentation de tous les éléments du paysage, a été levée en 1774. Elle montre pour le littoral de Sables d'Or une flèche dunaire largement développée et entièrement végétalisée, qui protège en arrière d’elle un marais maritime. Le développement de la station balnéaire de Sables d'Or a abouti à une urbanisation du massif dunaire en particulier au niveau de la flèche depuis les années 1970 .

Les évolutions de la végétation dunaire reportées sur la figure 3 (BERANGER, 2005) montrent qu'en 1952, l'extrémité du massif dunaire était entièrement couverte de végétation en bon état. En 1982, le front dunaire présente côté plage une végétation dégradée tout le long de l'extrémité de la flèche. Cette érosion, probablement liée à l'action de fortes houles lors des pleines mers de vive-eau, tend à créer des encoches en pied de dune qui déstabilisent le front dunaire peu végétalisé. Par ailleurs, toujours en 1982, les divagations du chenal de jusant à l'intérieur de la lagune provoquent des érosions du revers interne du massif dunaire.

En 2002, l'extrémité de la dune ne présente plus de dégradations importantes de la végétation, grâce aux aménagements visant à canaliser la fréquentation anthropique, ce qui permet à la végétation à oyats de stabiliser les sables dunaires. L'urbanisation de l'intérieur du massif dunaire, déjà bien établie en 2002, se retrouve en 2012 où des érosions s’observent sur le front dunaire externe côté plage mais également, pour la première fois, dans les secteurs internes de la flèche, côté lagune (figure 3).

L'évolution du front dunaire se traduit par : (i) un déplacement d'environ $50 \mathrm{~m}$ vers le Nord de l'extrémité du massif dunaire entre 1952 et 1982, avec un retrait latéral de 18 m, (ii) un déplacement de même ordre en sens inverse, vers le Sud, mais avec une érosion importante de $78 \mathrm{~m}$ vers l'Est entre 1982 et 2002 et enfin (iii) une stabilité de l'extrémité du massif entre 2002 et 2012, la végétation étant dégradée au contact de la haute plage et au niveau de la bordure interne de la dune côté lagune. 


\section{XIV ${ }^{\text {èmes }}$ Journées Nationales Génie Côtier - Génie Civil \\ Toulon, 29 juin au $1^{\text {er }}$ juillet 2016}
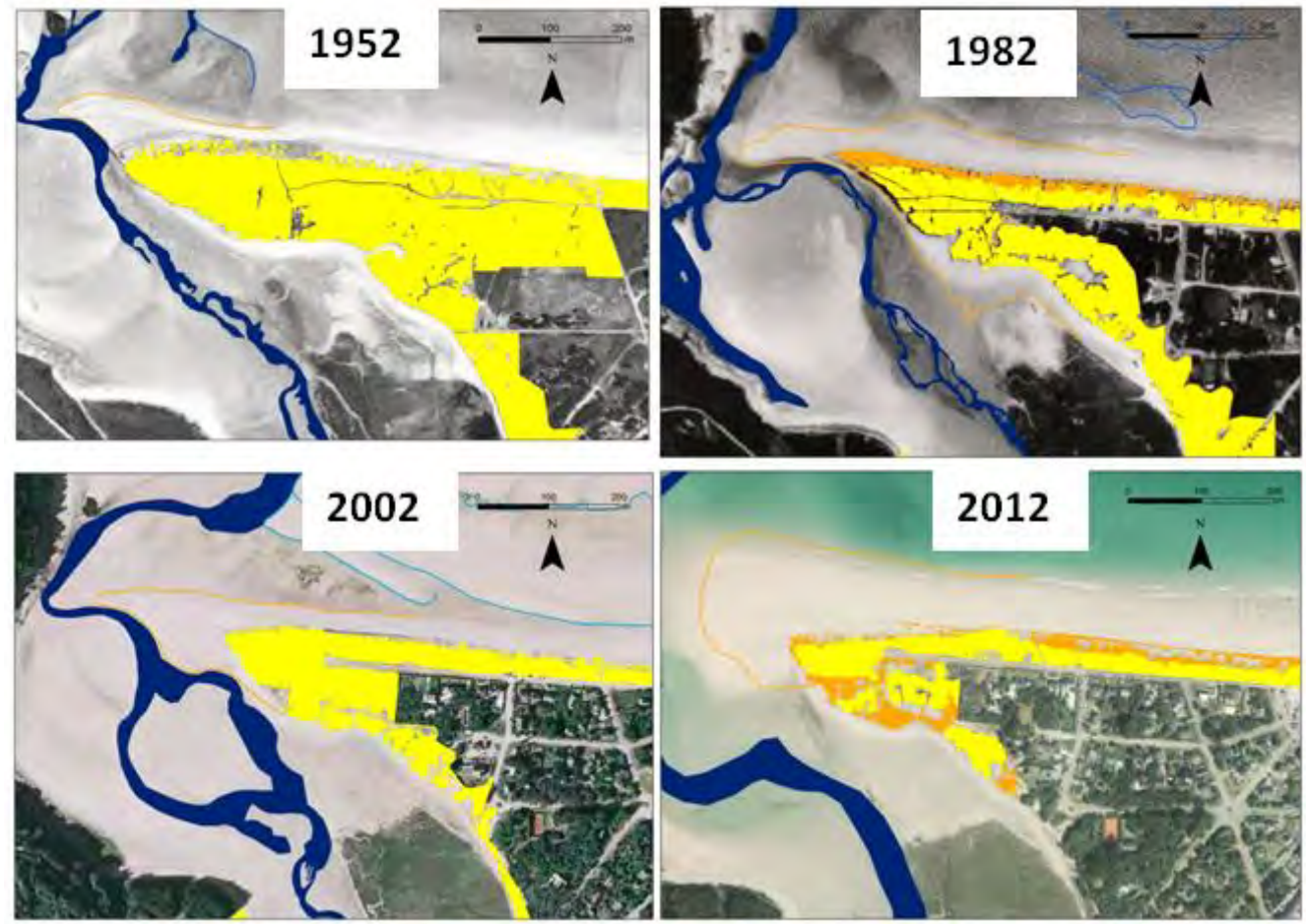

Figure 3. Evolution du massif dunaire entre 1952 et 2012

(en jaune : dune végétalisée, en orange : front dunaire dégradé, secteurs en érosion).

\subsection{Le schorre de la lagune}

Les parties les plus abritées de la lagune côtière sont occupées par un schorre qui couvre une surface d'environ 20 ha et dont l'évolution est relativement lente puisqu'il a progressé de 5 ha en 60 ans (figure 4). En 1952, le schorre couvrait 15,6 ha et 17,6 ha en 1982, soit une progression faible de la partie Est du schorre de 2 ha en 30 ans. Entre 1982 et 2002, c'est la partie Ouest qui a gagné 3 ha.

En 2002, le schorre continu couvre 20,5 ha et reste stable autour de 19,5 ha en 2012. Son développement ne s'effectue pas par une progression de sa bordure côté mer mais par une colonisation végétale plus continue de sa surface interne. La progression de la frange externe du schorre est freinée par l'envahissement des sables transportés et piégés à l’intérieur de la lagune.

\section{Discussion}

L'évolution de la morphologie de la flèche est étroitement liée aux échanges et transferts sableux entre les différentes structures morpho-sédimentaires que sont les barres sableuses de la plage externe, la flèche sableuse et son massif dunaire ainsi que la lagune comprenant le delta de flot et le schorre (BONNOT-COURTOIS et al., 2005). Ces transferts sont eux-mêmes conditionnés par les forçages naturels tels que la marée et les houles de tempêtes, la dérive littorale, les processus aériens et par des paramètres anthropiques tels que les aménagements et la fréquentation touristique (BODIN, 2015). 


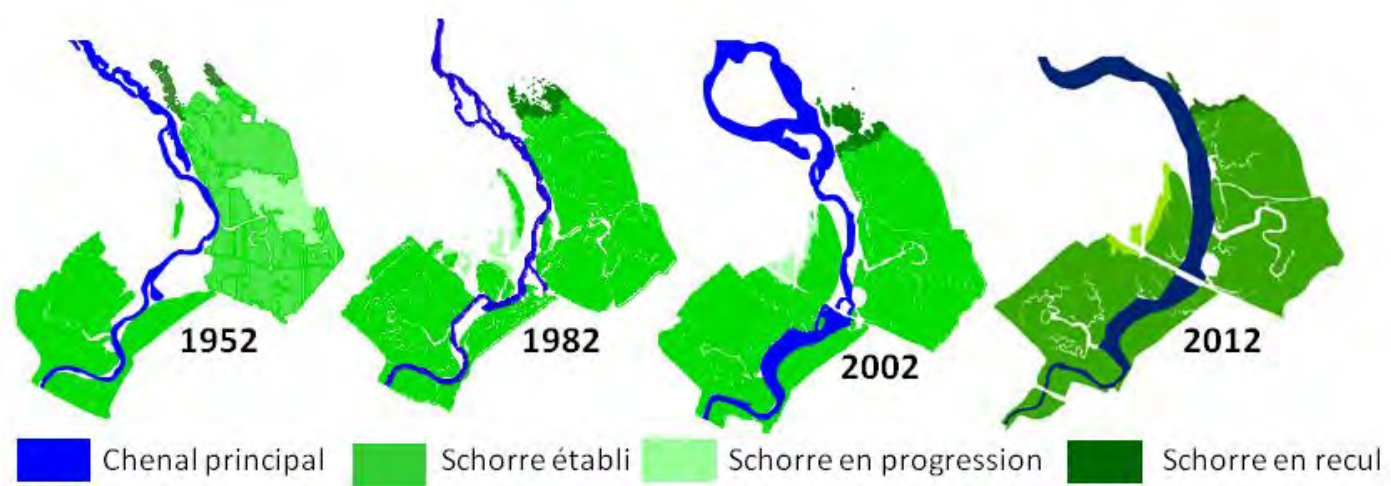

Figure 4. Evolution du schorre de la lagune de Sables d'Or depuis 1952.

Entre 1952 et 1982, la position de l'extrémité de la flèche sableuse reste stable, alors qu'entre 1982 et 2002, on note une extension ainsi qu'un étalement important vers l'Ouest de l'extrémité de la flèche. En 2012, la flèche semble retrouver sa position "initiale" de 1952. Cette flèche littorale est alimentée en sable non seulement par la dérive littorale venant de l'Est (figure 1) mais également à l'Ouest, par le système de barres et bâches qui se déplacent vers le haut estran depuis la basse et moyenne plage (BONNOT-COURTOIS et al., 2005). Ces deux processus dépendent de l'action de la houle, dépendante de son amplitude et de sa direction et donc des conditions de vent (figure 5). Le seuil de vitesse du vent de $8 \mathrm{~m} / \mathrm{s}$ est le seuil choisi pour les différents travaux de thèse réalisés à l’Université de Caen sur la façade de la Manche, seuil reconnu dans le Nord de la France (RUZ et al., 2009) et qui permet de comparer toutes ces études sur une base homogène. Entre 1952 et 1982, le nombre de jours de tempête par an varie alternativement de 5 à 35 jours, avec une baisse très nette des vents de secteur Nord/Nord-Est (0-90) de 1960 à 1982. Au contraire, de 1982 à 2012, la fréquence des tempêtes semble plus importante (17 jours/an), et notamment la fréquence des vents de secteur Nord-Est est plus régulière que lors de la période précédente (19521982). La dérive littorale, engendrée par ces houles est d'autant plus efficace pour le transport sédimentaire qu'elle est entretenue et peut ainsi expliquer l'extension vers l'Ouest de la flèche. De 2002 à 2012, la régression de la position de la flèche pourrait être corrélée à l'alternance de jours de tempêtes et de calme, diminuant l'énergie de la dérive littorale, favorisant ainsi l'érosion.

Les échanges de flux sableux entre le massif dunaire et l'extrémité de la flèche littorale semblent en équilibre depuis 1952. En effet, sur une période de 30 ans (1952-1982), la flèche s'est développée vers l’Ouest puis a retrouvé sa situation initiale. De 1982 à 2012, la flèche semble avoir suivi la même évolution, soit une extension vers l'Ouest suivi d'une régression vers sa position de 1982. Sur cette période, le retrait vers l'Est s'accompagne d'un étalement de la flèche, qui pourrait être imputé à l'accélération de la dégradation du massif dunaire entre 2002 et 2012. En effet, le sable n'étant plus fixé sur la dune, il peut être remobilisé alimentant le pied de dune puis la flèche. 


\section{XIV vèmes Journées Nationales Génie Côtier - Génie Civil \\ Toulon, 29 juin au $1^{\text {er }}$ juillet 2016}

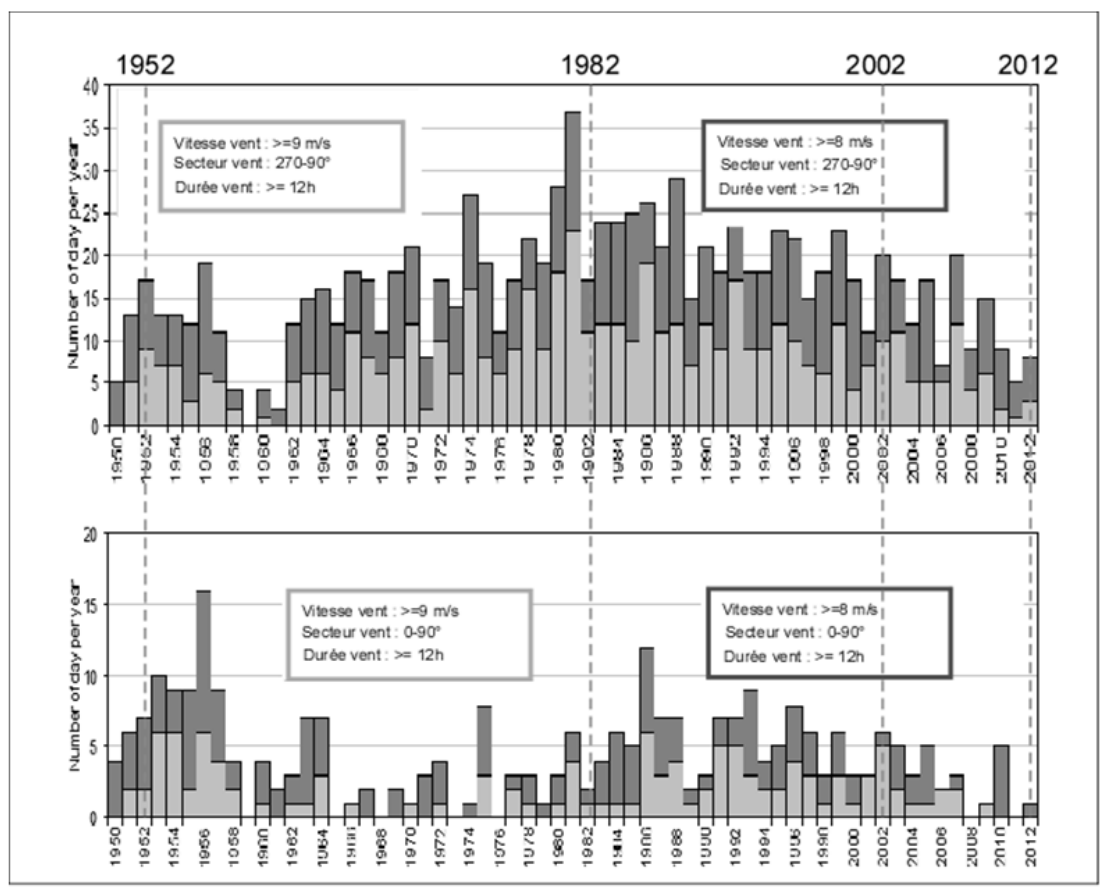

Figure 5.Occurrence des tempêtes, enregistrées à Dinard, de secteur 270-90 et 0-90.

Par ailleurs, les deux tiers de la surface occupée par la végétation naturelle du massif dunaire ont disparu entre 1952 et 2012. En effet, entre 1952 et 1966 la partie dunaire située sur le périmètre de l'ENS (Espace Naturel Sensible) s'est dégradée, tant à l’intérieur du massif que sur sa bordure externe. L'érosion éolienne est favorisée par la formation de brèches, elles-mêmes initiées par les nombreux chemins piétonniers dispersés dans la dune (figure 2 : 1982). Cependant, dès 1993, la végétation a recolonisé la dune, permettant de la fixer, grâce à la gestion de l'ENS qui a délimité des espaces piétonniers. Ainsi en 2002, le massif dunaire végétalisé s'est rétabli côté mer, mais il affiche une nette régression dans sa partie Sud, qui semble liée à l'extension de l'habitat urbain. En 2012, la bordure interne de la dune côté lagune est nettement dégradée, particulièrement à son extrémité où le chenal de jusant reprend les sables qui ne sont plus fixés par la végétation.

\section{Conclusion}

Depuis 60 ans, la morphologie globale de la flèche sableuse de Sables d'Or n'a pas subi de changements brusques les tempêtes étant suffisamment espacées pour que le système se cicatrise via une alimentation régulière en sable. Les transferts de sable s'établissent entre les différentes unités morpho-sédimentaires de ce littoral que sont le système barres/bâches, la flèche sableuse, le massif dunaire, le delta de flot et le schorre interne. Ainsi, la position de la flèche est similaire entre 1952 et 1982, puis de nouveau en 2012. Au sein de cette "cyclicité" de 30 ans, on observe entre 1982 et 2002 une importante 
extension vers l'Ouest de la flèche sableuse liée à une succession d'années caractérisées par une plus grande et régulière fréquence des tempêtes. En 2012, l'impact anthropique sur la végétalisation du massif dunaire accélère significativement la dégradation de ce massif, introduisant un "nouveau" transfert de sable, de la dune vers la flèche. La future évolution de ce site dépendra du déséquilibre induit par la dégradation du massif dunaire.

\section{Remerciements}

Les auteurs remercient sincèrement M. O. Le Bihan du Service des Espaces Naturels Sensibles au Conseil Général des Côtes d’Armor pour son soutien lors de cette étude.

\section{Références bibliographiques}

ALLEN H. (1982). Mesure de houle en différents sites du littoral français. EDF/LNH, Edition $n^{\circ} 5,18 \mathrm{p}+4$ figures et 424 planches.

BERANGER F. (2005). Evolution spatio-temporelle des estrans sableux et des fronts dunaires au Nord Est de la Baie de Saint-Brieuc. Stage de Master1, Lab. de Géom. et Env. Littoral. 23 p.

BODIN B. (2015). Etude géomorphologique du littoral de Sables d'Or les Pins. Rapport de stage (Capacités) pour le Département des Côtes d’Armor, 14 p.

BONNOT-COURTOIS C., LAFOND L.R. (1994). Dynamique sédimentaire du littoral de Sables d'Or les Pins (Côte d'Armor). Cahiers Nantais. Défense des côtes ou protection de l'espace littoral, $\mathrm{n}^{\circ}$ 41-42, pp 106-115.

BONNOT-COURTOIS C., LANÇON G. (2004).Erosion littorale, évaluation du risque. Phase 2 : Caractérisation de l'aléa érosion sur le littoral des Côtes d'Armor. Rapport DDE 22/UMR 8586 PRODIG CNRS. 29 p. + 19 Cartes et 47 tableaux.

BONNOT-COURTOIS C., BALTZER A., FOURNIER J. (2005). Complémentarité des photographies aériennes et des images sonar pour l'analyse des structures bio-morphosédimentaires du littoral. In : Milieux littoraux, Ed.L’Harmattan, pp 13-28.

LCHF (1982). Plage de Sables d'Or les Pins. Recherche des causes d'érosion. Rapport général LCHF, Commune de Fréhel. 30 p.

GLUARD L. (2012). Evolution des fonds sédimentaires sous l'influence de la divagation des chenaux aux abords du Mont St Michel. Thèse de doctorat, Univ. Caen. $313 \mathrm{p}$.

PLUET J., ZIMMERMANN C. (1984). Etude de la dynamique actuelle du littoral des Côtes du Nord du Cap d'Erquy au Cap Fréhel. Mémoire de Maîtrise Geogr. Phys. Université Paris VII, 113 p.

RUZ M. H., HEQUETTE A, MASPATAUD A. (2009). Identifying forcing conditions responsible for foredune erosion on the northern coast of France. Journal of Coastal Research. SI 56113 pp 356-360. 